\title{
HIGH-ENERGY FLARE EXPLOSIONS DRIVEN BY 3-DIMENSIONAL X-TYPE CURRENT LOOP COALESCENCE
}

\author{
Jun-ichi Sakai ${ }^{1}$ and Cornelis de Jager ${ }^{2}$ \\ 1 Department of Applied Mathematics and Physics \\ Faculty of Engineering, Toyama University \\ Toyama, 930 JAPAN \\ 2 SRON Laboratory for Space Research \\ Sorbonnelaan 2, 3584 CA Utrecht, The Netherlands
}

\begin{abstract}
We present a model for high-energy solar flare explosions driven by 3-dimensional X-type current loop coalescence. The 3-dimensional X-type loop coalescence, where two crossed flux: tubes interact in one point, is a fundamentally new process as compared to the 1-D and 2-D cases studied earlier. It is shown that, following the strong plasma collapse due to pinch effect, a point-like plasma explosion can be driven and also fast magnetosonic shock waves can be excited. We found that the conditions in the area producing the remarkable flare bursts of 21 May 1984 were indeed such that the many flare spikes could have been due to 3-D explosive X-type current loop coalescence. We also show, by studing the conding the conditions of shock formation in a Gamma ray flare, that the time delay of $\gamma$-rays from the impulsive phase could be the time needed for the shock formation in the flaring region.

We draw some general conclusions on the question why certain flares do emit $\boldsymbol{\gamma}$-rays in the Mev energy range, and why other, apparently important and large flares, do not. We accentuate the fact that a well-developed high-energy flare has three phases of particle acceleration.
\end{abstract}

\section{Introduction}

The current loop coalescence model of solar flares (Gold and Hoyle, 1960 ; Tajima, Brunel, and Sakai, 1982 ; Tajima et al., 1987 ; for a review see Sakai and Ohsawa, 1987) provides keys to understanding many of the characteristic of solar flares such as explosive plasma heating, high-energy particle (for both protons and electrons) acceleration, and quasi-periodic oscillation of electromagnetic emission. Recently it was shown (Sakai, 1989, 1990 ; Sakai and de Jagar, $1989 \mathrm{a}, 1899 \mathrm{~b})$ that the loop coalescence processes may have different signatures, depending on the geometry of the region containing the two interacting current loops. The key parameters characterizing different signatures are a scale L along the loop current, which characterizes the length of the interacting region of two loops, and a radius $\mathrm{R}$ of the current loop, as shown in Fig. 
1. As seen in Fig. 1 (a) where $\mathrm{L}>>\mathrm{R}$, an almost one-dimentional current sheet can be induced in the interacting region by the approach of two loops (Sakai and Ohsawa, 1987 ; Sakai, 1989). In this situation two current loops coalesce with periodic-osillations, when $B_{p}$ (magnetic field produced by the current) exceeds $B_{t}$ (magnetic field along the loop). During the coalecence both electrons and protons can be simultaneusly accelerated to relativistic energies within one second (Sakai, 1990). In contrast, when $\mathrm{B}_{p}<\mathrm{B}_{t}$, the loop coalesence proceeds associated with motions causing plasma tilting and without strong quasi-periodic oscillations.

When $\mathrm{L}>\mathrm{R}$, as seen in Fig.1(b), a flow of strong plasma jets can be driven by the plasma tilting motion around the magnetic reconnection point (Sakai, 1989). This mechanism has been applied to explain the coronal explosion (Sakai and de Jager, 1989b) .

The third situation where $\mathrm{L}<\mathrm{R}$, is the case of 3-dimensional X-type current loop coalescence (Sakai and de Jagar, 1989a), which we study in the present paper. The 3-

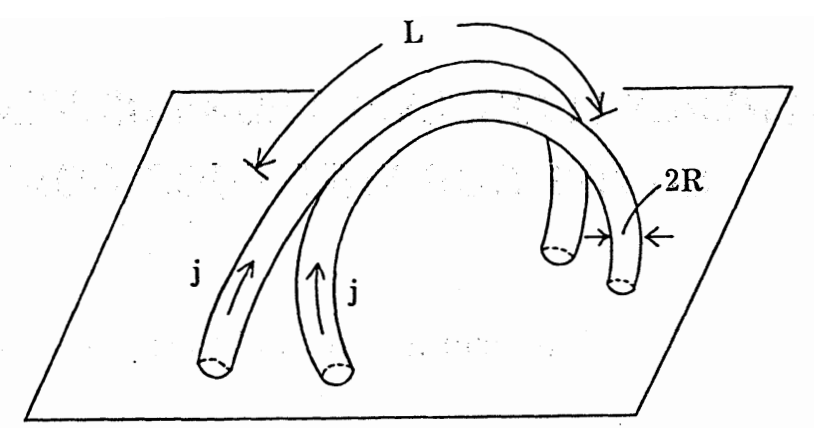

(a)

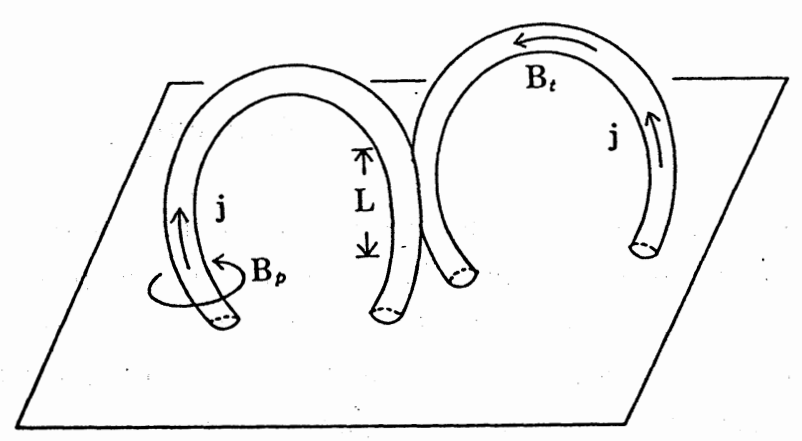

(b)

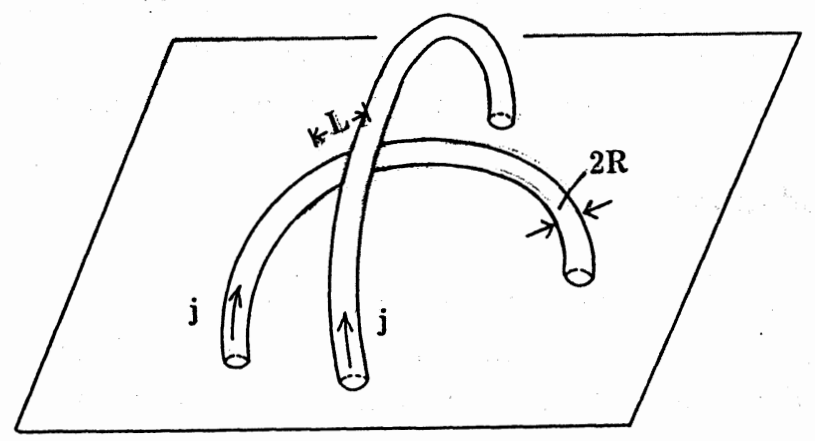

(c)

Fig. 1 Sehematic pictures showing three types of the current loop coalescence: (a) 1-D coalescence, (b) 2-D coalescence, (c) 3-D X-type coalescence. L is a characteristic length of the interacting region. $2 \mathrm{R}$ is a diameters of the loop with plasma current $\mathrm{j}$ along the magnetic field, $\mathrm{B}_{t} . \mathrm{B}_{p}$ is poloidal magnetic field produced by the plasma current. 
dimensional X-type current loop coalescence, where the two crossed flux-tubes interact in one point, is a fundamentally new process as compared with the above two cases. It is shown that a strong point-like plasma explosion can be driven by the plasma collapse due to the magnetic pinch effect, and also fast magnetosonic shock waves can be excited by the strong expanding plasma flows. We found that the conditions in the area producing the remarkable flare bursts of 21 May 1984 were due to 3-dimensional explosive X-type current loop coalecence. Table 1 summarizes some characteristics of three types of the current loop coalescence and typical solar flares explained by them.

We also found by studing the conditions of shock formation in a Gamma-ray flare that the time delay observed in high energy $\gamma$-rays could be identified with the time delay needed for the shock formation in the flaring region. In section 2 we present a theoretical model describing 3-dimentional X-type current loop coalescence, and we derive the basic equations. In section 3 we show some numerical results obtained from the basic equation. In section 4 we apply the 3-dimensional X-type current loop coalescence model to the 21 May 1984 flare. We also study the conditions of shock formation and discuss the time delay observed in high energy $\gamma$-rays, associated with high energy proton acceleration by shocks.

We draw some general conclusions on the question why certain flares do emit $\gamma$-rays in the Mev energy range, and why other, apparetly important and large flares, do not, We accentuate the fact that a well-developed high-energy flare has three phases of particle acceleration.

\section{Theoretical Model of 3-D X-type Coalescence}

As it is difficult to analyse the plasma dynamics in the whole region shown in Fig.1(c), we concentrate on the study of the local plasma behaviour near the X-type current interacting region.

Table 1

\begin{tabular}{|c|l|c|}
\hline 1-D & \multicolumn{1}{|c|}{$\begin{array}{c}\text { Characteristics of } \\
\text { Current Loop Coalescence }\end{array}$} & $\begin{array}{c}\text { Typical Solar Flares } \\
\text { and References }\end{array}$ \\
\hline Coalescence & $\begin{array}{c}B_{p}>B_{t}: \text { Quasi-periodic energy } \\
\text { release and high } \\
\text { energy particle } \\
\text { acceleration }\end{array}$ & $\begin{array}{c}1980 \text { June } 7 \\
1982 \text { Nov. } 26\end{array}$ \\
\hline 2-D & $\begin{array}{l}\text { Blasma jet formation driven by } \\
\text { tilting motion and shock } \\
\text { formation }\end{array}$ & $\begin{array}{c}\text { Sakai and Ohsawa } \\
\text { (19S7) }\end{array}$ \\
Coalescence & $\begin{array}{c}\text { (Coronal Explosion) } \\
\text { Sakai and de Jager } \\
\text { (19S9b) }\end{array}$ \\
\hline 3-D & $\begin{array}{l}\text { 3D point-like explosion } \\
\text { following strong magnetic } \\
\text { collapse and shock } \\
\text { formation }\end{array}$ & $\begin{array}{c}\text { 19S4 May 21 } \\
\text { Calescence }\end{array}$ \\
\hline
\end{tabular}


Recently, associated with the study of 3-dimensional steady magnetic reconnection near an $\mathrm{X}$-point magnetic configuration with an additional orthtogonal magnetic field component (Hesse and Schindler, 1988 ; Green, 1988; Sonneruup, 1988), Priest and Forbes (1989) showed that when 3-D magnetic reconnection occurs, there is a dynamic MHD behaviour with current concentration and strong plasma jets.

We start to assume that the 3-dimensional X-type cuurrent loop coalescence is essentially non-steady and dynamic process and the magnetic fields near the $\mathrm{X}$-point are given by

$$
\begin{aligned}
& B_{x}(y, t)=b_{x}(t) \frac{y}{\lambda}, \\
& B_{y}(x, t)=b_{y}(t) \frac{x}{\lambda}, \\
& B_{z}(t)=b_{z}(t),
\end{aligned}
$$

which are the lowest orders of expansion of merging-type magnetic fields close to the X-point (Hesse and Schindler, 1988). $\lambda$ is a characteristic scale-length of the X-type current interacting region. The time-dependent coefficents implying dynamic behaviour in Eq. (2-1) can be determined self-consistently from the MHD equations.

We also assume that the plasma flow velocities near the $\mathrm{X}$-point can be given by

$$
\begin{aligned}
& \mathrm{v}_{\mathrm{x}}(\mathrm{x}, \mathrm{y})=\frac{\dot{\mathrm{b}}}{\mathrm{b}} \mathrm{x}, \\
& \mathrm{v}_{\mathrm{y}}(\mathrm{y}, \mathrm{t})=\frac{\dot{\mathrm{a}}}{\mathrm{a}} \mathrm{y}, \\
& \mathrm{v}_{\mathrm{z}}(\mathrm{z}, \mathrm{t})=\frac{\dot{\mathrm{c}}_{1}}{\mathrm{c}_{1}} \mathrm{z},
\end{aligned}
$$

where we introduced three time-dependent scale factors, $a(t), b(t)$ and $c_{1}(t)$, which can be also determined later.

The dot means derivative with respect to time. We start from the following MHD equations,

$$
\begin{aligned}
& \frac{\partial \rho}{\partial \mathrm{t}}+\operatorname{div}(\mathrm{p} v)=0, \\
& \rho\left(\frac{\partial \mathrm{v}}{\partial \mathrm{t}}+\mathrm{v} \cdot \nabla \mathrm{v}\right)=-\nabla \mathrm{p}+\frac{1}{4 \pi} \operatorname{rot} \mathrm{Bx} B, \\
& \frac{\partial \mathrm{B}}{\partial \mathrm{t}}=\operatorname{rot}(\mathrm{v} \times \mathrm{B})+\frac{\mathrm{c}^{2}}{4 \pi \sigma} \triangle \mathrm{B}, \\
& \frac{\partial \mathrm{p}}{\partial \mathrm{t}}+\mathrm{v} \cdot \nabla \mathrm{p}+\gamma \operatorname{pdiv} \mathrm{v}=0,
\end{aligned}
$$

where we assumed that the process is adiabatic with adiabatic ratio, $\gamma$.

Substituting the expressions for velocities Eq. (2-2) into Eq. (2-3), we find that the plasma density $\rho(\mathrm{t})$ can be given by

$$
\rho(\mathrm{t})=\frac{\rho_{0}}{\mathrm{a}(\mathrm{t}) \mathrm{b}(\mathrm{t}) \mathrm{c}_{1}(\mathrm{t})},
$$

where $\rho_{0}$ is a constant.

By use of Eqs. (2-1) and (2-2), we obtain from Eq. (2-5) that the magnetic fields obey as follows,

$$
\mathrm{B}_{\mathrm{x}}(\mathrm{y}, \mathrm{t})=\frac{\mathrm{B}_{0}}{\mathrm{a}^{2} \mathrm{c}_{1}} \frac{\mathrm{y}}{\lambda},
$$




$$
\begin{aligned}
& \mathrm{B}_{\mathrm{y}}(\mathrm{x}, \mathrm{t})=\frac{\mathrm{B}_{0}}{\mathrm{~b}^{2} \mathrm{c}_{1}} \frac{\mathrm{x}}{\lambda}, \\
& \mathrm{B}_{\mathrm{z}}(\mathrm{t})=\frac{\mathrm{B}_{\mathrm{z} 0}}{\mathrm{ab}},
\end{aligned}
$$

where $\mathrm{B}_{0}$ and $\mathrm{B}_{\mathbf{}}$ are constants.

If we assume that the plasma pressure $p(x, y, z, t)$ can be expressed as

$$
\mathrm{p}(\mathrm{x}, \mathrm{y}, \mathrm{z}, \mathrm{t})=\mathrm{p}_{0}(\mathrm{t})-\mathrm{p}_{\mathrm{x} 0}(\mathrm{t}) \frac{\mathrm{x}^{2}}{\lambda^{2}}-\mathrm{p}_{\mathrm{y} 0}(\mathrm{t}) \frac{\mathrm{y}^{2}}{\lambda^{2}}-\mathrm{p}_{\mathrm{z} 0}(\mathrm{t}) \frac{\mathrm{z}^{2}}{\lambda^{2}}
$$

we find from Eq. (2-6) that the time-dependent coefficients in Eq. (2-9) can be given by

$$
\begin{aligned}
& \mathrm{p}_{0}(\mathrm{t})=\frac{\mathrm{p}_{00}}{\left(\mathrm{abc}_{1}\right)^{\gamma},} \\
& \mathrm{p}_{\mathrm{x} 0}(\mathrm{t})=\frac{\mathrm{p}_{00}}{\left(\mathrm{ac}_{1}\right)^{\gamma} \mathrm{b}^{\gamma+2}}, \\
& \mathrm{p}_{\mathrm{y} 0}(\mathrm{t})=\frac{\mathrm{p}_{00}}{\mathrm{a}^{\gamma+2}\left(\mathrm{bc}_{1}\right)^{\gamma}}, \\
& \mathrm{p}_{\mathrm{z} 0}(\mathrm{t})=\frac{\mathrm{p}_{00}}{(\mathrm{ab})^{\gamma} \mathrm{c}_{1}^{\gamma+2}},
\end{aligned}
$$

where $\mathrm{p}_{00}$ is a constant.

Finally we obtain the basic equations describing the time evolution of three shale factors, a $(t), b(t)$, and $c_{1}(t)$ from the equatins of motion, Eq. (2-4) as

$$
\begin{aligned}
& \frac{\mathrm{d}^{2} \mathrm{a}}{\mathrm{dt}^{2}}=\frac{\beta_{\mathrm{p}}}{\mathrm{a}^{\gamma}\left(\mathrm{bc}_{1}\right)^{\gamma-1}}+\frac{1}{\mathrm{c}_{1}}\left(\frac{1}{\mathrm{~b}}-\frac{\mathrm{b}}{\mathrm{a}^{2}}\right), \\
& \frac{\mathrm{d}^{2} \mathrm{~b}}{\mathrm{dt}^{2}}=\frac{\beta_{\mathrm{p}}}{\left(\mathrm{ac}_{1}\right)^{\gamma-1} \mathrm{~b}^{\gamma}}-\frac{1}{\mathrm{c}_{1}}\left(\frac{\mathrm{a}}{\mathrm{b}^{2}}-\frac{1}{\mathrm{a}}\right), \\
& \frac{\mathrm{d}^{2} \mathrm{c}_{1}}{\mathrm{dt}^{2}}=\frac{\beta_{\mathrm{p}}}{(\mathrm{ab})^{\gamma-1} \mathrm{c}_{1}^{\gamma}},
\end{aligned}
$$

where time is normalized by $\tau_{\mathrm{A}}=\lambda / \mathrm{v}_{\mathrm{AO}}$, where $\mathrm{v}_{\mathrm{AO}}=\mathrm{B}_{\mathrm{o}} / \sqrt{4 \pi \rho_{0}}$, and $\beta_{\mathrm{p}}$ (poloidal plasma beta ratio depending on the amplitude $\mathrm{B}_{0}$ of magnetic fields produced by the plasma current) is defined as

$$
\beta_{\mathrm{p}}=\frac{8 \pi \mathrm{p}_{00}}{\mathrm{~B}_{0}^{2}}=\left(\frac{\mathrm{B}_{\mathrm{zo}}}{\mathrm{B}_{0}}\right)^{2} \beta, \text { where } \beta=\frac{8 \pi \mathrm{p}_{00}}{\mathrm{~B}_{z 0}^{2}},
$$

In Eqs, (2-11)-(2-13), the first terms at the right-handed side show the plasma pressure gradient, while the second terms show the magnetic Lorentz force, causing the plasma pinch effect, which can become strong when the plasma $\beta$ is small.

The plasma current induced by the X-type coalescence flows almost along the $z$-direction and can be given by

$$
\mathrm{j}_{z}(\mathrm{t})=\frac{\mathrm{cB} \mathrm{B}_{0}}{4 \pi \lambda \mathrm{c}_{1}}\left(\frac{1}{\mathrm{~b}^{2}}-\frac{1}{\mathrm{a}^{2}}\right),
$$

Once the three time-dependent scale factors can be determined from the above equations, we find all physical quantities from them.

\section{Numerical results}

\subsection{3-D point-like explosion following strong magnetic collapse}

We show here some numerical results obtained from Eqs; (2-11)-(2-13). The important parameter which controls the plasma dynamics near the $\mathrm{X}$-type coalescence region is the poloidal 
plasma beta $\beta_{\mathrm{p}}$ in Eqs. (2-11)-(2-13). If $\beta_{\mathrm{p}}$ is less than about one, we observe strong plasma compression (magnetic collapse) driven by the magnetic pinch effect in the direction of coalescence.

This strong magnetic collapse can continue to drive strong point-like 3-dimensional explosion. On the other hand, when $\beta_{\mathrm{p}}$ is larger than about one, we only observe weak plasma expansion without plasma collapse. The critical $\beta_{\mathrm{p}}$, depending on whether the strong plasma collapse can occur or not, is about $\beta_{\mathrm{p}}=1.4$, as appears from the numerical calculation.

We show results for the following initial conditions;

$$
\mathrm{a}=1, \dot{\mathrm{a}}=-0.1, \mathrm{~b}=2, \dot{\mathrm{b}}=0, \mathrm{c}_{1}=1, \dot{\mathrm{c}}_{1}=0 \text { and } \beta_{\mathrm{p}}=0.5 \text {, }
$$

where $\mathrm{v}_{\mathrm{y}}(\mathrm{t}=0)=\dot{\mathrm{ax}} / \mathrm{a}=-0.1 \mathrm{x}$ means that the colliding direction of the two current loops is the $\mathrm{y}$-direction. We note that the results are insensitive to the initial colliding velocity. The adiabatic ratio $\gamma$ is taken to be $\gamma=5 / 3$.

Figure 2 shows the time history of plasma velocity components. The strong plasma inflow $\left(\mathrm{v}_{\mathrm{y}}<0\right)$ toward the $\mathrm{X}$-point can be driven by the magnetic pinch effect. After the maximum in the collapsing flow, which occurs around $0.87 \tau_{\mathrm{A}}$, the plasma motion rapidly changes into an outward expansion. This phenomenon is a point-like plasma explosion driven by the magnetic plasma collapse. During the magnetic collapse, the plasma density enhances by about a factor 10 as compared with the initial density $\rho(t=0) / \rho_{0}=0.5$, as seen in Fig. 3.

Figure 4 shows the time history of the magnetic field components. As a consequence of the strong plasma collapse an almose one dimensional current sheet $\left(B_{x}>>B_{y}\right)$ can be transiently formed near the $\mathrm{X}$-point. The magnetic field, $\mathrm{B}_{z}$ almost along the current loop can enhance by a factor of about 10 as compared with the initial strength, $B_{z}(t=0) / B_{z 0}=0.5$.

Figure 5 shows time history of plasma pressrure. As seen in the figure, the plasma pressure, $\mathrm{p}_{\mathrm{y} 0}$ in the collapsing direction can be strongly enhanced.

We note that during the strong magnetic collapse the magnetic field can change drastically, which can lead to the generation of strong inductive electric fields. These strong electric fields can accelerate particles to high energy within a very short period of time.

Figure 6 shows the time history of plasma velocities when $\beta_{\mathrm{p}}=1.47$. As seen in the figure of $\mathrm{v}_{\mathrm{y}}$, there occurs a very weak plasma inflow, which is associated with very weak plasma expansion.

Therefore, we conclude from these numerical results that when the poloidal plasma $\beta_{\mathrm{p}}$ is less than about one, the strong magnetic plasma collapse can drive a violent $3-\mathrm{D}$ point-like plasma explosion within a very short interval of time.

We make note about the results oftained for $\gamma=5 / 3$. When $\gamma=5 / 3$, the basic equations $(2-$ $11)-(2-13)$ seem to be explosive near the implosion time $t=0.87$. Therefore the results after $\mathrm{t}=0.87$ are apparent. Near the implosion time, the current sheet becomes nearlly one dimen: sional strcture. Therefore if we use $\gamma=3$ near the implosion time, we have the explosions for $\mathrm{V}_{\mathrm{y}}$ as shows in Fig.2.

We need more investigation for the explosion dynamics near the implosion time.

\subsection{Fast magnetosonic shock wave formation}

We found in the previous section that strong outward plasma flow can be rapidly driven by 
the magnetic collapse. Here we examine the possibility whether fast magnetosonic shock waves can be excited or not by the strong expanding plasma flow.

The shock formation can occur when the local plasma flow velocity exceeds the local magnetosonic velocity, namely

$$
\mathrm{v}^{2}>\mathrm{c}_{\mathrm{s}}^{2}+\mathrm{v}_{\mathrm{A}}^{2}
$$

This condition can be written as follow by use of khysical expressions for $\mathrm{v}, \mathrm{B}, \rho, \mathrm{p}$ that appeared in the previous section;

$$
\begin{aligned}
& {\left[\left(\frac{\mathrm{b}}{\mathrm{b}}\right)^{2}-\frac{\mathrm{a}}{\mathrm{b}^{3} \mathrm{c}_{1}}+\frac{\beta_{\mathrm{p}}}{\left(\mathrm{ac}_{1}\right)^{\gamma-1} \mathrm{~b}^{\gamma+1}}\right] \tilde{\mathrm{x}}^{2}+} \\
& {\left[\left(\frac{\mathrm{a}}{\mathrm{a}}\right)^{2}-\frac{\mathrm{b}}{\mathrm{a}^{3} \mathrm{c}_{1}}+\frac{\beta_{\mathrm{p}}}{\mathrm{a}^{\gamma+1}\left(\mathrm{bc}_{1}\right)^{\gamma-1}}\right] \tilde{\mathrm{y}}^{2}+} \\
& {\left[\left(\frac{\mathrm{c}_{1}}{\mathrm{c}_{1}}\right)^{2}+\frac{\beta_{\mathrm{p}}}{\left(\mathrm{ac}_{1}\right)^{\gamma+1} \mathrm{c}^{\gamma+1}}\right] \tilde{\mathrm{z}}^{2}>} \\
& \frac{\mathrm{c}_{1}}{\mathrm{ab}}+\frac{\beta_{\mathrm{p}}}{\left(\mathrm{abc}_{1}\right)^{\gamma-1}},
\end{aligned}
$$

where $\tilde{x}=\mathrm{x} / \lambda, \tilde{\mathrm{y}}=\mathrm{y} / \lambda$, and $\tilde{z}=z / \lambda$.

The following time-dependent coefficients, $\mathrm{X}_{\mathrm{s}}, \mathrm{Y}_{\mathrm{s}}$, and $Z_{\mathrm{s}}$ are crucial for the shock formation ;

$$
\begin{aligned}
& \mathrm{X}_{\mathrm{s}}=\left(\frac{\mathrm{b}}{\mathrm{b}}\right)^{2}-\frac{\mathrm{a}}{\mathrm{b}^{3} \mathrm{c}_{1}}+\frac{\beta_{\mathrm{p}}}{\left(\mathrm{ac}_{1}\right)^{\gamma-1} \mathrm{~b}^{\gamma+1}}, \\
& \mathrm{Y}_{\mathrm{s}}=\left(\frac{\mathrm{a}}{\mathrm{a}}\right)^{2}-\frac{\mathrm{b}}{\mathrm{a}^{3} \mathrm{c}_{1}}+\frac{\beta_{\mathrm{p}}}{\mathrm{a}^{\gamma+1}\left(\mathrm{bc}_{1}\right)^{\gamma-1}} \\
& \mathrm{Z}_{\mathrm{s}}=\left(\frac{\mathrm{c}_{1}}{\mathrm{c}_{1}}\right)^{2}+\frac{\beta_{\mathrm{p}}}{(\mathrm{ab})^{\gamma-1} \mathrm{c}^{+1}} .
\end{aligned}
$$

If $X_{s}$ and $Y_{s}$ are positive, then the shock waves can be produced. Figure 7 shows the time history of the above defined quantities $X_{s}$, and $Y_{s}$. As seen in the figure, fast magnetosonic shock waves can be generated after the strong plasma magnetic collapse. The fast magnetosonic shock waves can also quite rapidly accelerate protons to relativistic energies. These may then emit highenegy $\boldsymbol{\gamma}$-rays (Sakai and Ohsawa, 1987). The acceleration time to relativistic energies
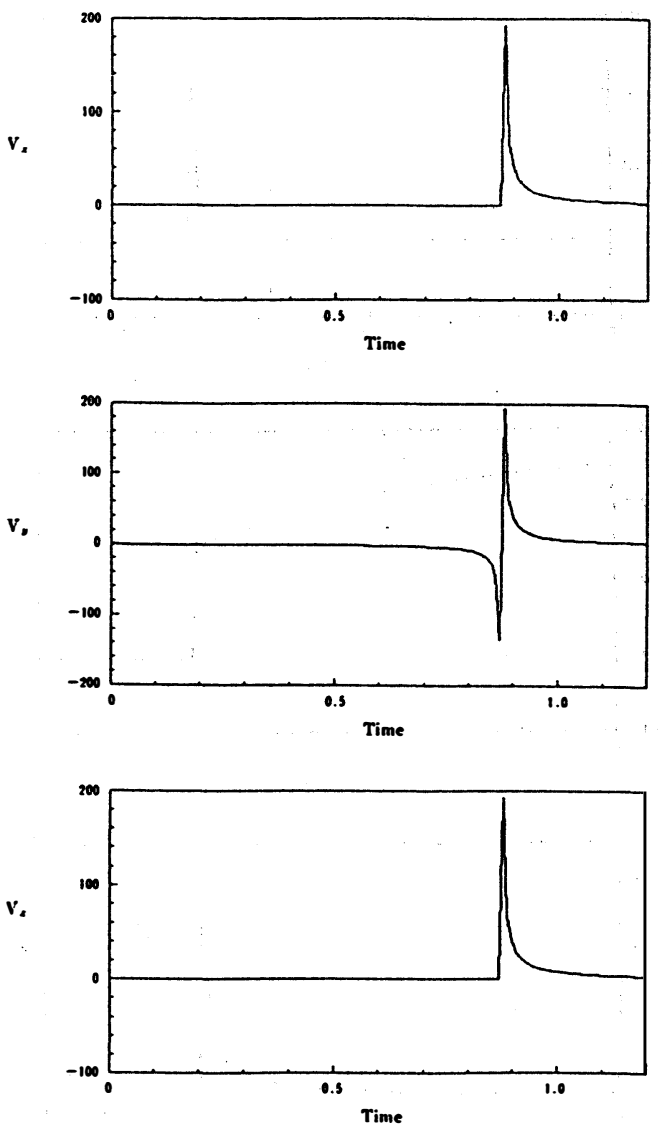

Fig. 2

Fig. 2 Time history of three components of the plasma velocity $\mathrm{v}_{x}=\mathrm{b} / \mathrm{b}, \mathrm{v}_{y}=\mathrm{a} / \mathrm{a}, \mathrm{v}_{z}=\mathrm{c}_{1} / \mathrm{c}_{1}$ for $\beta_{p}=0.5$. The time is normalized by $\tau_{\mathrm{A}}$, and the velocity is normalized by $\mathrm{v}_{\mathrm{AO}}$. The strong plasma inflow $\left(\mathrm{v}_{y}<0\right)$ occurs in a coalesing direction, after then strong pointlike explosion occurs in all diretions.

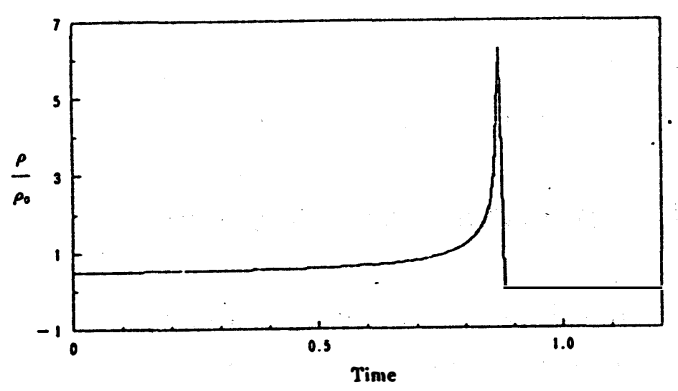

Fig. 3 Time history of the plasma density $(\rho /$ $\left.\rho_{0}\right)=\left(\mathrm{abc}_{1}\right)^{-1}$ for $\beta_{p}=0.5$. The time is normalized by $\tau_{\mathrm{A}}$ and the density is normarized by $\rho_{0}$. The initial density is $0.5 \rho_{0}$. 

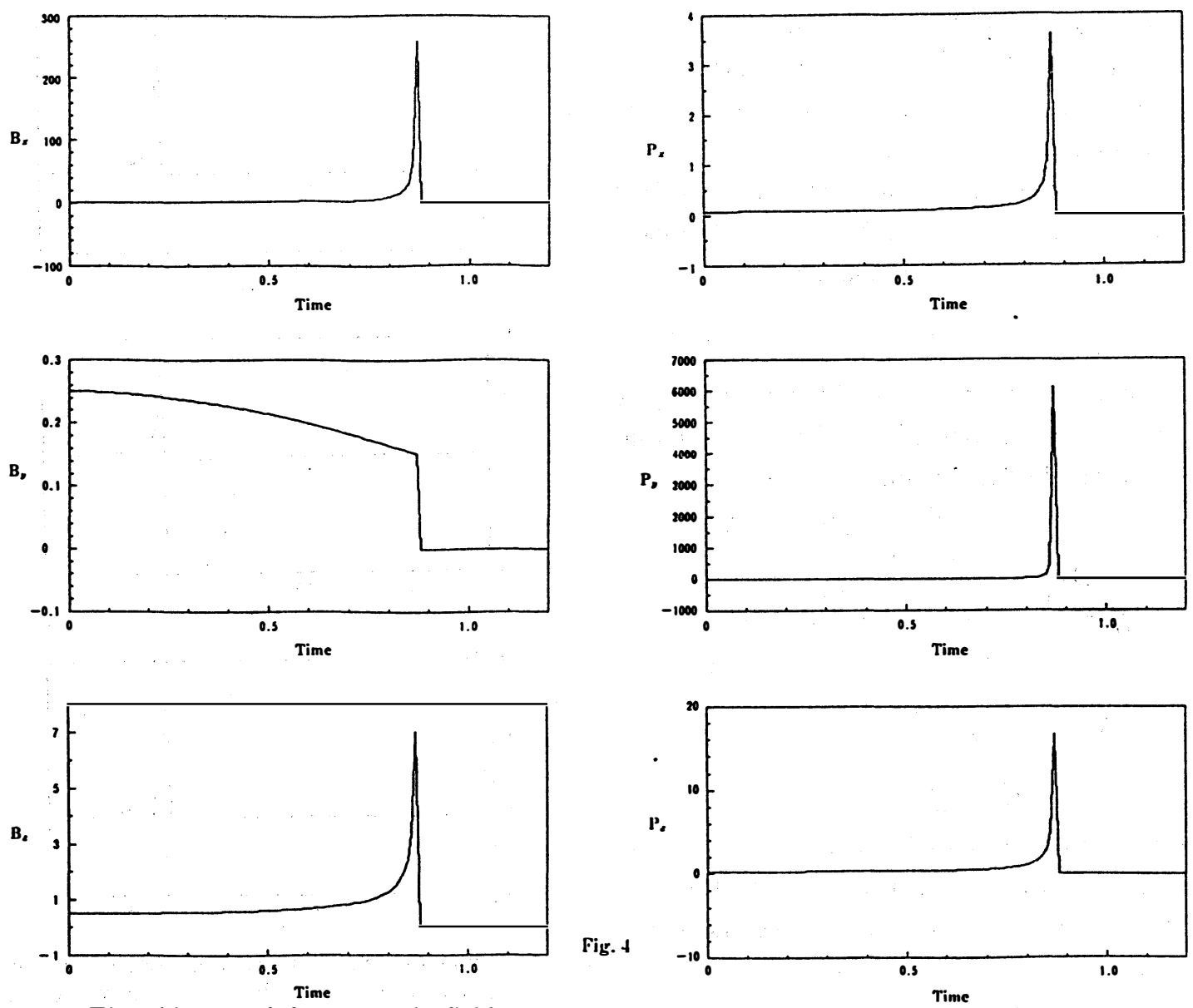

Fig. 4 Time history of the magnetic field components for $\beta_{p}=0.5$. The time is normalized by $\tau_{\mathrm{A}}$. $\mathrm{B}_{\mathrm{x}}=\left(\mathrm{a}^{2} \mathrm{c}_{1}\right)^{-1}$ and $\mathrm{B}_{\mathrm{y}}=\left(\mathrm{b}^{2}\right.$ $\left.\mathrm{C}_{1}\right)^{-1}$ are normalized by $\mathrm{B}_{0}$, and $\mathrm{B}_{z}=$ $(\mathrm{ab})^{-1}$ is normalized by $\mathrm{B}_{z 0}$.

Fig. 4

Fig. 5 Time history of the plasma pressures for $\beta_{p}=0.5$. The time is normalized by $\tau_{\mathrm{A}}$ and the pressures are normalized by $\mathrm{p}_{00}$.

by fast magnetosonic shocks is quite short and less than one second. Therefore it becomes very important for the explanation of the observed time delays in solar Gamma ray emmision to know how fast the fast magnetosonic shock waves can be formed in a flaring region. We will discuss this problem in the next section.

\section{Applications and discussion}

In this section we wish to apply the foregoing results to a few, mostly high-energy, flares and we draw some general conclusions on the question why certain flares do emit in the Mev energy range, and why other, apparently important and large flares, do not. . We accentuate the fact that well-developed high-energy flare has three phases of particle acceleration.

\subsection{The small energetic flare of May $211984 ; 13: 26$ UT}

This flare, observed by Kaufmann et al. $(1985,1986)$ was remarkable in many respects. It 
lasted for about a minute only, and consisted of a number of impulsive bursts. The most remarkable of these was one at $13: 26: 32$ UT. It lasted for three seconds only, but at millimeter waves $(90 \mathrm{GHz}$, a surprizingly high frequency for flare radiation) it was the strongest burst of the flare and the same was true in $\mathrm{X}$-rays of energies above $100 \mathrm{Kev}$. Very remarkable was the fine structure in microwaves. At $90 \mathrm{GHz}$ the burst consisted of at least 13 discrete spikes, each with an average duration of about $0.1 \mathrm{~s}$. The bursts were almost symmetric, with rise and decay times of $60 \mathrm{~ms}$.

This 3-seconds burst complex was discussed by Kaufmann et al. (1986), de Jager et al. (1987) and Sakai and de Jager (1989a). In the second of these studies it was shown that the radiation properties of each of the 0 . $1 \mathrm{~s}$-bursts could be explained quantitatively by the following course of events. By some mechanism plasma is accelerated to energies up to several $100 \mathrm{Kev}$, with a kinetic temperature of about $5 \times 10^{8} \mathrm{~K}$, and an electron density of $10^{11} \mathrm{~cm}^{-3}$. The linear size of this plasma knot is about $350 \mathrm{~km}$. Its magnetic field is 1400 to $2000 \mathrm{G}$. It can then be shown that such a plasma knot will loose its high energy by collisionless conduction in about 50 $\mathrm{ms}$, a value very close to the observed decay times of each of the $0.1 \mathrm{~s}$ bursts. What apparently happened here was that by 13 successive processes of nearly explosive energy injections 13 flare knots were successively formed, each in less than about $60 \mathrm{~ms}$. Each of these knots radiated the observed intensities in X-rays and microwaves with the observed spectral characteristics, and conductively lost its energy in the observed burst decay time of $60 \mathrm{~ms}$.

The processes described above leave us with the following five questions: (a) Can
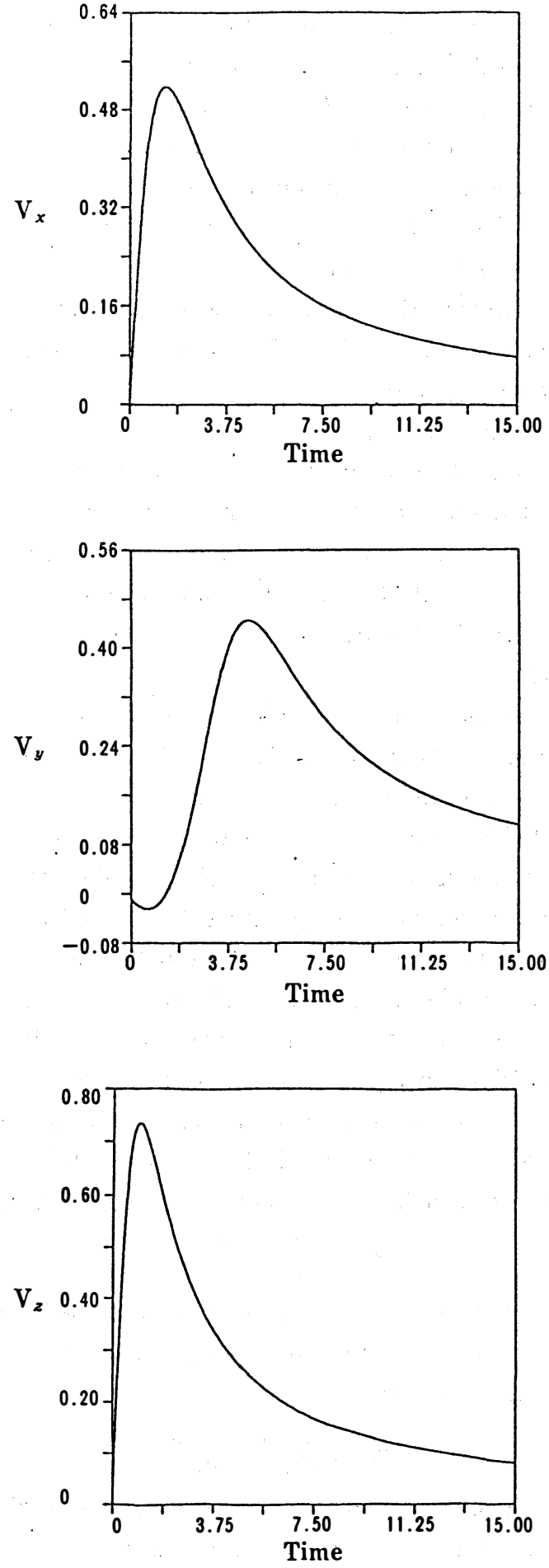

Fig. 6 Time history of the plasma velocities for $\beta_{p}=1.47$. The time is normalized by $\tau_{\mathrm{A}}$ and the velocities are normalized by $\mathrm{v}_{\mathrm{AO}}$. 
we visualize an acceleration mechanism that causes the observed degree of energization in a time as short as $60 \mathrm{~ms}$ ? (b) Is it allowed to speak of the plasma knot 'temperature', in other words, were the plasma knots thermal? (c) Were only electrons accelerated or ions too, and if so to what energies? (d) How to explain that there were 13 successive energy injection? (e) Why did this burst complex not emit gamma rays?

The first question was answered by Sakai and de Jager (1989a) who demonstrated that these knots can originate by process of 3-D X-type flux-tube coalescence. Below we will show that the observations can be explained fairly well in a quantitative way,

A spherical region with a diameter of 350 $\mathrm{km}$ and a magnetic field B of 1400 to $2000 \mathrm{G}$ would contain a magnetic energy $\mathrm{B}^{2} \mathrm{~V} / 8 \pi$ of 2 to $4 \mathrm{X} 10^{27} \mathrm{erg}$. The thermal energy content of that volume, assuming an electron density $\mathrm{n}_{\mathrm{e}}=10^{11} \mathrm{~cm}^{-3}$ and temperature $\mathrm{T}=5 \times 10^{8} \mathrm{~K}$, is $1.5 \times 10^{26} \mathrm{erg}$. Hence the magnetic energy og the region is sufficient to provide of each of the flare spikes. In order to know if conditions in the flaring region are suitable
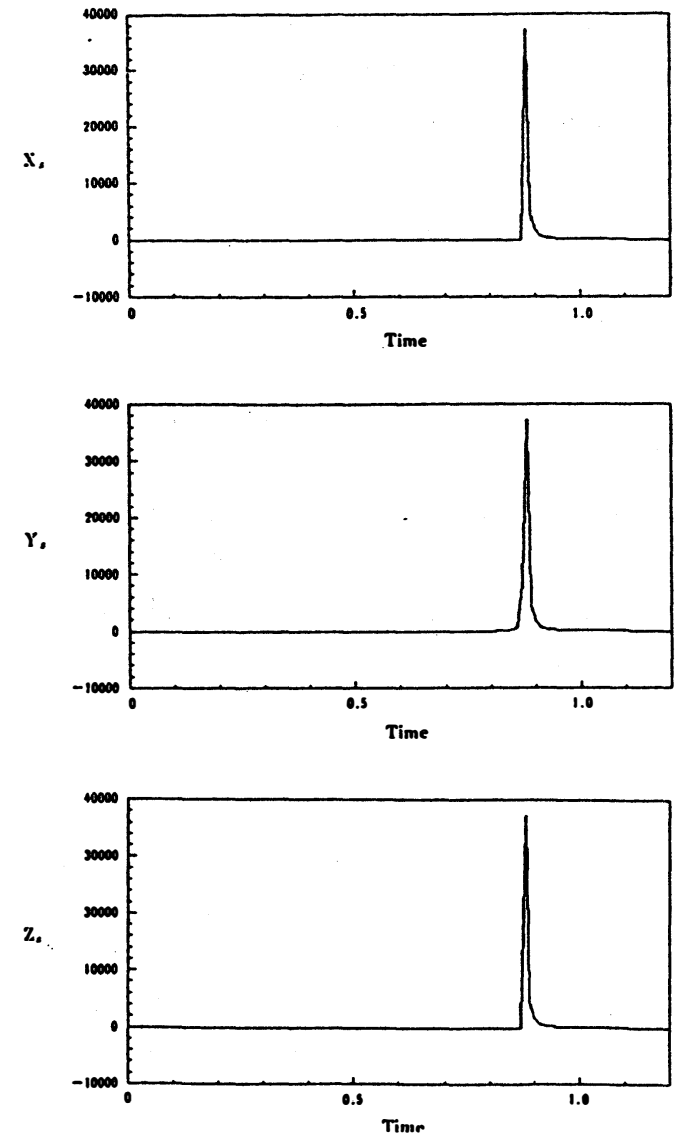

Fig. 7 Time history of the expressions defined in the text; $X_{s}, Y_{s}, Z_{s}$. The fast magnetosonic shock waves can be produced when $X_{S}$ and $Y_{S}$ are positive $\left(Z_{S}\right.$ is always positive). for the explosive coalescence one has to know the value of the poloidal plasma beta $\beta_{\mathrm{p}}$ before coalescence, as defined in Fq. (2-14). On the other hand, we can obtain the value of the plasma beta, $\beta_{\mathrm{a}}$, which depends on the magnetic field, $\mathrm{B}_{\mathrm{z}}$ along current loop after coalescence as follows ;

$$
\beta_{\mathrm{a}}=\frac{8 \pi \mathrm{p}_{\mathrm{a}}}{\mathrm{B}_{2}^{2}}=\frac{8 \pi \mathrm{p}_{00}}{\mathrm{~B}_{\mathrm{z0}}^{2}} \frac{\mathrm{a}^{2} \mathrm{~b}^{2}}{\left(\mathrm{abc}_{1}\right)^{\gamma}},
$$

where we used $\mathrm{p}_{\mathrm{a}}=\mathrm{p}_{00} /\left(\mathrm{abc}_{1}\right)^{\gamma}$ for the pressure (see Eq. (2-10)) and $\mathrm{B}_{z}=\mathrm{B}_{z 0} / \mathrm{ab}$ for the magnetic field (see Eq. (2-8)) after coalescence. The above expression for $\beta_{\mathrm{a}}$ can be rewritten by the use of Eq. (2-14) as

$$
\beta_{\mathrm{a}}=\left(\frac{\mathrm{B}_{0}}{\mathrm{~B}_{\mathrm{z} 0}}\right)^{2} \frac{\beta_{\mathrm{p}}}{(\mathrm{ab})^{\gamma-2} \mathrm{c}_{1}^{\gamma}},
$$

which relation connects the two plasma beta's : $\beta$ after coalescence and $\beta_{\mathrm{p}}$ before coalescence. This relation can be also rewritten as

$$
\beta_{\mathrm{a}}=\left(\frac{\mathrm{B}_{0}}{\mathrm{~B}_{\mathrm{z} 0}}\right)^{2}\left(\frac{\mathrm{B}_{\mathrm{z} 0}}{\mathrm{~B}_{z}}\right)^{2}\left(\frac{\rho}{\rho_{0}}\right)^{5 / 3} \beta_{\mathrm{p}},
$$

where we used the compression ratios of the magnetic field and densities of after and before 
coalescence.

The condition for explosive 3-D X-type coalescence, $\beta_{\mathrm{p}}<1.4$ gives us an upper limit, $\beta_{\mathrm{au}}$ of $\beta_{\mathrm{a}}$ as

$$
\beta_{\mathrm{a}}<1.4\left(\frac{\mathrm{B}_{0}}{\mathrm{~B}_{\mathrm{z} 0}}\right)^{2}\left(\frac{\mathrm{B}_{\mathrm{z} 0}}{\mathrm{~B}_{\mathrm{z}}}\right)^{2}\left(\frac{\rho}{\rho_{0}}\right)^{5 / 3} \equiv \beta_{\mathrm{au}} .
$$

Using $B=1500 \mathrm{G}, \mathrm{n}=10^{11} \mathrm{~cm}^{-3}$ and $\mathrm{T}=5 \times 10^{8} \mathrm{~K}$, the plasma beta, $\beta_{\mathrm{a}}$ for the above flare becomes $\beta_{\mathrm{a}}=0.01$, If we take the vale of $\beta_{\mathrm{p}}=0.5$, as suggested from the considerations in the previous section, we obtain $\mathrm{B}_{z 0} / \mathrm{B}_{z} \simeq 0.1, \rho / \rho_{0} \simeq 10$. Therefore we get

$$
\beta_{\mathrm{a}}=0.23\left(\frac{\mathrm{B}_{0}}{\mathrm{~B}_{\mathrm{z} 0}}\right)^{2},
$$

From $\beta_{\mathrm{a}}=0.01$ and Eq. (4-5), we find that the magnetic field, $\mathrm{B}_{0}$ produced by the loop current before coalescence was $B_{0}=0.2 \times B_{z 0}$ is the magnetic field along the loop before coalescence. As $\mathrm{B}_{\mathrm{z} 0}=0.1 \times \mathrm{B}_{\mathrm{z}}=0.1 \times 1500 \mathrm{G}=150 \mathrm{G}$, we find $\mathrm{B}_{0}=30 \mathrm{G}$.

From $B_{0}=30 G$ and $n_{0}=10^{10} \mathrm{~cm}^{-3}$, we get $v_{A 0}=B_{0} / \sqrt{4 \pi \rho_{0}}=660 \mathrm{~km} / \mathrm{s}$. With this value of $V_{A 0}$, we find $\tau_{\mathrm{A}}=\lambda / \mathrm{v}_{\mathrm{A} 0}=0.53 \mathrm{~s}$, if we take $\lambda=350 \mathrm{~km}$ for the flaring region. With the numerical result $\beta_{\mathrm{p}}=0.5$, we find from the numerical data shown in Figure 2 an explosion time of about $0.05 \tau_{\mathrm{A}}$, which corresponds to about $26 \mathrm{~ms}$. This explosion time is very close to observed value of above $60 \mathrm{~ms}$.

The explosion velocity after coalescence is about $200 \mathrm{v}_{\mathrm{A} 0}$, which corresponds to $1.2 \times 10^{10} \mathrm{~cm} /$ $\mathrm{s} \sim \mathrm{c} / 3$. This predicted explosion velocity yields electron energies of about $100 \mathrm{KeV}$, as observed. We therefore conclude that the observed short acceleration time is indeed predicted by the mechanism of current loop coalescence, as well as the observed electron energies.

The answer to the second question can be derived as follows (cf. Lang, 1974, p.225). Electrons, injected into a coronal plasma with a velocity of $\mathrm{c} / 3$ have a collision time of $0.17 \mathrm{~s}$; three times the observed acceleration time of the bursts. In other words, one-third of electrons involved would become thermalized in the acceleration time. We would obtain a defletion time of $50 \mathrm{~ms}$ if the velocity of injection was only slightly shorter, $70000 \mathrm{~km} / \mathrm{s}$.

The answer is therefore that the electron component of the plasma is marginally thermalized. The answer to the third question is that the ionic gas is not thermalized because the deflection time for ions is a million times longer than for electrons, and the electron-ion collision time in the given conditions is $2 \mathrm{~s}$. Scince after $2 \mathrm{~s}$ the plasma has already lost its energy (the conduction loss time is $60 \mathrm{~ms}$ ), the ionic gas cannot be heated. The answer to the fourth question, why there are 13 explosions, may be given by a model proposed by one of us (de Jager, 1986, 1988) : There are strong indications that in the pre-flare plasma there exist many thin fluxtubes, which we prefer to call fluxthreads (the 'spaghetti bundle'). Among these, many interactions may occur when the topology of the area changes, for example by motions in the footpoint area and by the disturbances produced by the explosive 3-D coalescence at one locality. Here, we should note that for another efficient physical successive triggering mechanisms in surrounding regions, interactions between finite amplitude fast magnetosonic waves and current sheets (Sakai and Washimi, 1982; Sakai, 1982, 1983 ; Sakai et al., 1984) can drive explosive magnetic reconnection.

These answers also explain why this burst complex was not observed in Gamma-rays. To that end ionic acceleration is needed to $10^{7}$ or $10^{8} \mathrm{eV}$, which may happen in a shocked medium of 
sufficiently large size (see the next subsection). The conditions in the flare discussed here were apparently not sufficient to create such particle energies.

\subsection{Shock formation in a gamma flare}

From the observations by SMM and Hinotori satellites, it became clear that there are two classes of gamma-ray/proton (GR/P) flares-; impulsive GR/P flares and gradual GR/P flares (see for reviews, Bai and Sturrock, 1989 ; Yoshimori, 1989). The GR/P flares refer to flares that produce nuclear gamma-rays and /or energetic interplanetary protons. Most of short duration flares $(<100 \mathrm{~s}$ ) corresponds to the impulsive GR/P flares, while most of long duration flares ( $>$ $200 \mathrm{~s})$ corresponds to the gradual GR/P flares.

In the impulsive GR/P flares there are two phases of particle accelerations ; first phase is that both electrons and protons are accelerated to high energies at the same time (within one second) (Kane et al. 1986), and second phase is that electrons up to tens of $\mathrm{MeV}$ within from a few seconds to $100 \mathrm{~s}$ are accelerated, and also protons are accelerated to Gev energies. The second phase acceleration is associated with gamma-rays peak delay from hard X-ray peak of the first impulsive phase. The gamma-ray peak time delay is in from $2 \mathrm{~s}$ to about $100 \mathrm{~s}$. From the gamma-ray time delay Bai et al. proposed the second step acceleration. For this second step acceleration various mechanisms, mainly involving shocks (Decker and Vlahos, 1986 ; Ellison and Ramaty, 1985 ; Ohsawa and Sakai, 1988) have been proposed.

As shown by Ohsawa and Sakai (1988), simultaneous electron and proton acceleration to relativistic energies can be achieved by fast magnetosonic shock waves in a relatively high magnetic field. The acceleration time to relativistic energies is quite short and much less than one second (Ohsawa and Sakai, 1988). This shock acceleration mechanism can correspond to the above second phase of the impulsive GR/P flares. Then a question what the observed gamma-ray peak time delays mean is proposed. Once the fast magnetosonic shock waves can be produced from the flaring regions, it is sufficient to explain the observed results, because the acceleration time is quite short. The finite shock formation time in the flaring regions can correspond to the gamma-ray peak delay time.

The fast magnetososic shock formation time is given by

$$
\tau_{\text {shock }} \sim \mathrm{L} / \mathrm{v}_{\mathrm{A}},
$$

where $\mathrm{L}$ is a characteristic length of disturbances of the flaring region, and $\mathrm{v}_{\mathrm{A}}$ is the Alfvèn velocity. If we assume that the gamma-ray peak time delay, $\tau_{\text {delay }}$ corresponds to the above shock formation time, $\tau_{\text {shock }}$, the gamma-ray delay is propotional to the characteristic flaring scale, $\mathrm{L}$. If we use $\mathrm{L}=10^{7}-10^{10} \mathrm{~cm}$ and $\mathrm{v}_{\mathrm{A}}=10^{8} \mathrm{~cm} / \mathrm{s}$, the gamma-ray delay time is about $0.1-$ $100 \mathrm{~s}$, which is in the observed value (Yoshimori, 1989). Yoshimori (1989) showed from the Hinotori observation that the gamma-ray delay time is proprtional to the flare duration time as well as the $\mathrm{H}_{\alpha}$ importance. This result is consistent with the above assumption that the observed gamma-ray delay is time needed for the shock formation in the flaring region. The flaring region is of the same order as the diameters of the interacting current flux tubes.

In order to be able to detect the gamma-rays, there is a threshold for the number of accelerated protons; $8 \times 10^{31}$ for $\mathrm{E}>30 \mathrm{MeV}$ (Chupp, 1984). This means that even if the small 
energetic flare like the 21 May 1984 flare can produce the gamma-rays, it can not be observed.

\subsection{Three phases of acceleration in high-energy flares}

One of us (de Jager, 1988) has forwarded the hypothesis that in high-energy flares three phases of particle acceleration may occur. Two of these have been discussed in this paper: First phase: Rapid X-type coalescence of two flux tubes, yielding an electron plasma with electron energies of the order of 100 to $1000 \mathrm{KeV}$. A high-energy plasma knot can originate at the place of the current loop interaction. The size of the plasma knot is comparable to the diameters of the flux tube. As seen in the 1-D explosive coalescence (Sakai, 1989), high energy protons producing the line gamma-rays can be also generated by the strong electric fields during the magnetic collapse. This simultaneous acceleration of both electrons and protons may also operate in the 3-D X-type coalescence, because of generation of strong electric fields during the magnetic collapse.

Second phase: Formation of a field of shock waves in the plasma knot formed in the first phase. The fast magnetosonic shock waves can rapidly accelerate both electrons and protons to relativistic energies by the strong coherent electric field in the shock front (Ohsawa and Sakai, 1988). This second phase follows the first after a brief period, which delay can be observed in the gamma-rays peak delay. The delay is about one or two seconds, but also there are cases in which it lasts as long as a minute. This delay time can be explained by the time needed for the fast magnetosonic shock formation in the flaring regions.

The third phase has not been discussed in this paper, but the ideas about it were around for many years already. Usually, this last phase is denoted in literature as the second step or second phase of acceleration (Wild et al., 1963 ; de Jager, 1969). We prefer to call it the third case, for obvious reasons. Ramaty and Murphy. (1987) have found new and in our views conclusive support for it by stressing and supporting with quantitative arguments that the high energy particles, whth energies up to GeV's, observed in interplanetary space after energetic solar flares, and which are apparently formed tens of minutes after the impulsive of flares are accelerated in the shock wave medium that forms after such flares, in field line systems opening to space.

\section{Acknowledgements}

One of us (J.S.) would like to thank The Netherlands Research Organisation (N.W.O) for a visiting fellowship. He acknowledges the hospitality of Prof. J.A.M. Bleeker at the Laboratory for Space Research in Utrecht, where a part of this work began. He would like to thank Dr. M. Yoshimori for his discussion about the Hinotori gamma-rays results.

\section{References}

Bai, T., Kiplinger, A.L., and Dennis, B.R. : 1984, Bull. Am. Astron. Soc. 16, 535.

Bai, T., and Sturrock, P.A. : 1989, Annu. Rev. Astron. Astrophys. 27, 421.

Chupp, E.L. : 1984, Annu. Rev. Astron. Astrophys. 22, 359. 
Decker, R.B., and Vlahos, L. : 1986, Ap. J. 306, 710.

De Jager, C. : 1969, In Solar Flares and Space Research, Proc. Symp. Plenary Meet. COSPAR, 11th pp. 1-15. Amsterdam, North-Holland, 419.

De Jager, C. : 1986, Space Sci. Rev. 44, 43.

De Jager, C. : 1988, 'Energetic Phonomena in Impulsive Solar Flares', in '20th Intern. Cosmic Ray Conf.' Nauka, Moscow, Vol.7, 66.

De Jager, C., Kuijpers, J., Correia, E., and Kaufmann, P. : 1987 Solar Phys. 110, 317

Ellison, E.C., and Ramaty, R. : 1985, Ap. J. 298, 400.

Gold, T., and Hoyle, F. : 1960, Monthly Notices Roy. Astoron. Soc. 120, 89.

Green, J.M. : 1988, J. Geophys. Res. 93, 8583.

Hesse, M., and Schindler, K. : 1988, J. Geophys. Res. 93, 5559.

Kane, S.R., Chupp, E.L., Forrest, D.J., Share, G.H., and Riger, E. : 1986, Ap. J. Left. 300, L95.

Kaufmann, P., Correia, E., Costa, J.E.R., Zodi Vaz, A.M., and Dennis, B.R. : 1985, Nature, 313, 380.

Kaufmann, P., Correia, E., Costa, J.E.R., and Zodi Vaz, A.M. : Lang, K.R. : 1974, 'Astrophysical Quantities' Springer, Berlin

Ohsawa, Y., and Sakai, J-I. : 1988, Ap. J. 332, 439.

Priest, E.R., and Forbes, T. : 1989, Solar Phys. 119, 211.

Ramaty, R., and Murphy, R.J. : 1987, Space Sci. Rev. 45, 213.

Sakai, J-I. : 1982, Ap. J. 263, 970.

Sakai, J-I. : 1983, J. Plasma Phys. 30, 109.

Sakai, J-I. : 1989,Solar Phys. 120,117.

Sakai, J-I. : 1990, Ap. J. Suppl. 73, June issue.

Sakai, J-I., and de Jager, C. : 1989a, Solar Phys. 123, 389.

Sakai, J-I., and de Jager, C. : 1989b, Solar phys. 123, 393.

Sakai, J-I., and Ohsawa, Y. : 1987, Space Sci. Rev.46, 113.

Sakai, J-I., Tajima, T., and Brunel, F. : 1984, Solar Phys. 91, 103.

Sakai, J-I., and Washimi, H. : 1982, Ap. J. 258, 823.

Sonnerup, B.U.O. : 1988, Computer Phys. Commun. 49, 143.

Tajima, T., Brunel, F., and Sakai, J-I. : 1982,Ap. J. Lett. 245,L45.

Tajima, T., Sakai, J-I., Nakajima, H., Kosugi, T., Brunel, F., and Kundu, M.R. : 1987, Ap. J. 321, 1031.

Yoshimori, M. : 1989, Space Sci. Rev. 51, 85.

Wild, J.P., Smerd, S.F., and Weiss A.A.: 1963, Annu. Rev. Astron. Astrophys. 1, 291. 